\title{
Comparison of Common Image Gathers from Kirchhoff and Fresnel Zone Paraxial Kirchhoff Migrations
}

Manuel J. S. Costa, Universidade Federal do Pará - UFPA

João Carlos R. Cruz, Universidade Federal do Pará - UFPA
Copyright 2019, SBGf - Sociedade Brasileira de Geofísica

This paper was prepared for presentation during the $16^{\text {th }}$ International Congress of the Brazilian Geophysical Society held in Rio de Janeiro, Brazil, 19-22 August 2019.

Contents of this paper were reviewed by the Technical Committee of the $16^{\text {th }}$ International Congress of the Brazilian Geophysical Society and do not necessarily represent any position of the SBGf, its officers or members. Electronic reproduction or storage of any part of this paper for commercial purposes without the written consent of the Brazilian Geophysical Society is prohibited.

\begin{abstract}
This work considers 2D true amplitude modified Kirchhoff type prestack depth migration in the common offset domain. The appoach considers the superposiion of paraxial amplitudes in the vicinity of every trace location in a common offset section, mapped throgh the determination of the Fresnel zone elements of the seismic experimente. These amplitudes are the stacked and smeared by the use paraxial operador, which is a highfrequency representation of the seismic wave field, prior to being migrated in th common offset domain to is true position in the depth domain.
\end{abstract}

\section{Introduction}

Imaging methods applied to seismic reflection data focus their tasks on collapsing diffractions tails and correctly positioning reflection events to their true depth positions in the Earth. For hydrocarbon exploration thus, considering that an acurrate velocity model is available via data processing, the main objective is to produce the best possible images of subsurface reflectors of interest to help interpreters in mapping structural interfaces that sepa rate oil-bearing geologic formations and that may represent traps for oil and gas accumulations. Among the seismic imaging methods used by oil indus try, Kirchhofftype methods and wave-equation-based methods are the most representative, each one with its own advantages and disadvantages. These different imaging methods may be applied to certain classes of sorted do mains: poststack and prestack, 2D or 3D. The final result (i.e., subsurface migrated images) may be displayed in depth or in time. The method to be studied in this paper refers to a Kirchhoff-type, 2D prestack depth migration. Among the methods cited above, Kirchhoff migration (Schneider, 1978; Bleistein, 1987; Schleicher et al., 1993; Tygel et al., 1996) is extensively used to obtain subsurface images from seismic data. This is due to, among others, its low computational cost when compared to others methods, especially in 3D, its high reliability, besides the fact that it can handle several kinds of wavefields. Kirchhoff migration is highly versatile, since it is possible to equally migrate and image large to small datasets, including wavefield datasets with different acquisition or parameterization geometries. In this sense, considering the continuous need to improve the resolution of seismic images, the present work deals with an integral solution of the Kirchhoff migration applied to a prestack depth 2D migration dataset. The proposed integral migration operator was be modified in order to introduce in its kernel a paraxial wavefield approximation represented by a beam stack. This approximation, in our approach, works in the same manner as a Green function of the imaging problem in the common-offset domain (Ferreira and Cruz, 2009; Costa, 2012), at the same time considering the use of a true-amplitude migration theory (Schleicher et al., 1993; Tygel et al., 1996) in order to attenuate the effects of the geometrical spreading on the reflection amplitudes. In this work we present a qualitative comparison of the images obtained (common - image gathers and sections migrated) using a true-amplitude 2D Kirchhoff depth migration operator (K-PSDM) and a trueamplitude 2D Kirchhoff modified depth migration operator (KM-PSDM), in the common-offset domain.

\section{True-amplitude 2D Kirchhoff migration theory (K- PSDM)}

The general form of the Kirchhoff type migration integral in 2D is given by (Schleicher et al., 1993; Tygel et al., 1996)

$$
I(R)=\int_{A} d \xi w(\xi, R) D\left[U\left(\xi, t=\tau_{D}(\xi, R)\right)\right]
$$

in which $I(R)$ denotes the stacked amplitude (output) assigned to the diffraction point at $R$ in depth-migrated space, $U(\xi, t)$ represents the (input) seismic data, sorted in an specific configuration and described by the configuration parameter $\xi$, wehre the variation of the configuration parameter occurs inside the migration aperture $A$ and weighted by function $w(\xi, R)$. The operator $D$ represents a half-derivative applied to the data to restore the wavelet shape and $\tau_{D}(\xi, R)$ determining the traveltime the diffraction curve (Huygens curve). 


\section{D Integral superposition of paraxial contributions}

The procedure to be considered here is the simulation of the seismic wavefield by a superposition of paraxial contributions, where the wavefield is generated by a source point inside a medium and decomposed into contributions for each individual ray. In this sense, following the migration theory proposed in Ferreira e Cruz (2005), Sun et al. (2000), Costa (2012) and Garabito et al. (2012) the kernel of the migration integral (1) is modified by a superposition of contributions in the vicinity of a specular reference ray, through a stacking along the parabolic curve (see, 6) confined to a size determined by the projected Fresnel zone $\left(H_{P}\right)$, where it is calculated by (Costa, 2012):

$$
H_{P}=\frac{\Lambda^{2}}{H_{F}}
$$

of which

$$
\Lambda=\left(-\frac{Q_{2, R G}}{\cos \beta_{G} \cos \beta_{S}}\right)^{-1} \Gamma_{G}+\left(-\frac{Q_{2, S R}}{\cos \beta_{G} \cos \beta_{S}}\right)^{-1} \Gamma_{S}
$$

and

$$
H_{F}=\frac{Q_{1, R G}}{Q_{2, R G}} \cos ^{2} \beta_{S}+\frac{P_{2, S R}}{Q_{2, S R}} \cos ^{2} \beta_{G}
$$

In this approach, $\Gamma_{S}, \Gamma_{G}$ are scalars to be specify depending on the seismic configuration, $\beta_{S}$ and $\beta_{G}$ define the angles that the central ray forms with the normal along the acquisition line, $P_{2}, Q_{1}$ e $Q_{2}$ scalar elements that characterize the central ray, which come from of the dynamic ray tracing system, $S R$ and $R G$ are indexes that specify each ray branch. Therefore, the internal stacking for a given diffraction time $\tau_{D}$ of the reference trace $\xi_{D}$ belonging to a local window of input data $U(\xi, t)$ is defined as follows:

$$
\hat{U}\left(\xi_{D}, \tau_{D}\right)=\int_{A P} d \xi_{p} \sqrt{H_{P}\left(\xi_{P}\right)} L\left(\xi_{P}\right) U\left(\xi_{P}, T_{P}\left(\xi_{P}, \xi_{D}\right)\right)
$$

where

$$
T_{P}\left(\xi_{P}, \xi_{D}\right)=\tau_{D}\left(\xi_{D}\right)+p\left(\xi_{P}-\xi_{D}\right)+\frac{1}{2} H_{P}\left(\xi_{P}\right)\left(\xi_{P}-\xi_{D}\right)^{2}
$$

represents the relative parabolic curve that collects the paraxial contributions, $\quad p$ the slowness horizontal of the central ray, $\tau_{D}\left(\xi_{D}\right)$ the traveltime of the diffraction curve (Huygens curve), $L\left(\xi_{P}\right)$ the decay of the seismic data amplitude inside the stacking centered on the reference coordinate. Aperture $A_{P}$ is the equivalent of the size of the projected Fresnel zone towards the acquisition surface (Schleicher et al., 1997; Schleicher et al., 2004), defined around the reference trace $\xi_{D}, \xi_{P}$ represents the position of the points in the vicinity of the reference seismic trace. Finally, $\sqrt{H\left(\xi_{P}\right)}$ determines the weight function of the internal stacking inside the projected Fresnel zone.

\section{Modified True-amplitude 2D Kirchhoff migration theory (KM-PSDM)}

Considering the 2D Kirchhoff migration integral defined previously (1) and the definition of the paraxial contributions superposition integral given by (5), where the latter modifies the kernel of (1), we arrive at the final modified Kirchhoff migration integral. In this new approach, the seismic data is primarily determined by a superposition of paraxial contributions as in (5). Without loss of generality, we then have in the common-offset (CO) configuration (Costa, 2012):

$$
\begin{aligned}
& I(R)=-\frac{1}{2 \pi} \int_{A} d x_{m} w\left(x_{m}, R\right) \int_{A_{P}} \sqrt{H_{P}\left(x_{m}^{P}\right)} D_{L}\left(x_{m}^{P}\right) \\
& D^{*}\left[U\left(x_{m}, t=\tau_{D}\left(x_{m}, x_{m}^{P}, R\right)\right)\right]
\end{aligned}
$$

where $I(R)$ is the migrated amplitude assigned to one diffraction point $R$ in depth, $U\left(x_{m}, t=\tau_{D}\left(x_{m}, x_{m}^{P}, R\right)\right)$ denotes the input data for the beam stacking defined by the second operator integral in (7). In this sence, the result of this second integral is the input data to be migrated through the $\tau_{D}(\xi, R)$ of the first operator integral, $x_{m}$ represents the position of the reference trace to be migrated, $x_{m}^{P}$ denots the position of the points in the vicinity of the reference trace within the aperture $A_{P}$, $\sqrt{H_{P}\left(x_{m}^{P}\right)}$ weight function of the beam stacking inside the projected Fresnel zone, $D_{L}\left(x_{m}^{P}\right)$ explicit a taper function, Finally, $D^{*}$ is the duble half time-derivative operator.

\section{KM-PSDM prestack depth migration algorithm: main steps}

In this section the steps that implement the migration $\mathrm{KM}$ $A C$ are listed:

I - Input:

- CO data

II- For each image point of the output section, calculate:

- The midpoint, the half-offset, the CO diffraction curve and the weight function associated with the $\mathrm{CO}$ diffraction curve. 
III- Diffraction curve times:

- For each time of the CO diffraction curves, calculete the $\mathrm{CO}$ paraxial curve;

- Parameters that define the aperture and weight the superpositions of paraxial contributions, after that sum the paraxial traces;

IV- stacking the amplitudes:

- Stacking the amplitudes coming from of the paraxial sum along the of $\mathrm{CO}$ diffraction curve, repeating the procedure for all sampling points of the output sections;

V- Output:

- Sections migrated to each common-offset; Stack the migrated gathers.

\section{Numerical results}

In this section we compare the migration results of the Kirchhoff $(\mathrm{K})$ and modified Kirchhoff $(\mathrm{KM})$ procedures by means of the common image gathers (CIGs) and sections migrated, respectively. The synthetic data used was the Marmousi, which consists of 261 common-shot (CS) gathers with 96 traces each. The shot interval is $25 \mathrm{~m}$ and the interval between traces is $25 \mathrm{~m}$. The near and far offsets are $100 \mathrm{~m}$ and $2475 \mathrm{~m}$. The total recording time is $3 \mathrm{~s}$, while the sampling interval is $4 \mathrm{~ms}$. The original Marmousi velocity model is shown in Figure (1). In Figure 2 (a) and Figure 2 (b) we compare the K-PSDM (see, 1) and KM-PSDM (see, 7) migrations, respectively. The image resulting by the KM-PSDM algorithm is cleaner because it has fewer artifacts than the one obtained by the Kirchhoff operator. Finally, as a way to verify the sensitivity of the migrations to the errors present in the velocity model, we will visualize common image gathers (CIGs) along the half offset dimension in Figure 3 (a) and Figure 3(b). Similarly, the main difference is in the final resolution, and it is also evident that the moveout errors in the KM-PSDM CIGs due to velocity inaccuracies are much more attenuated than in the CIGs of K-PSDM.

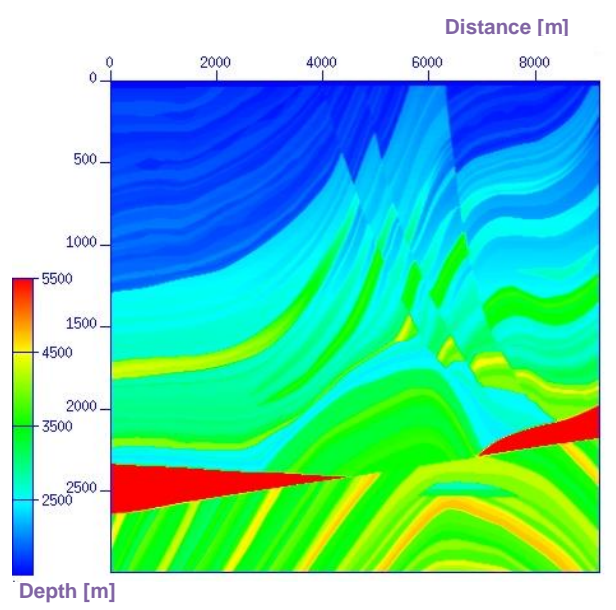

Figure 1 - Marmousi velocity model.
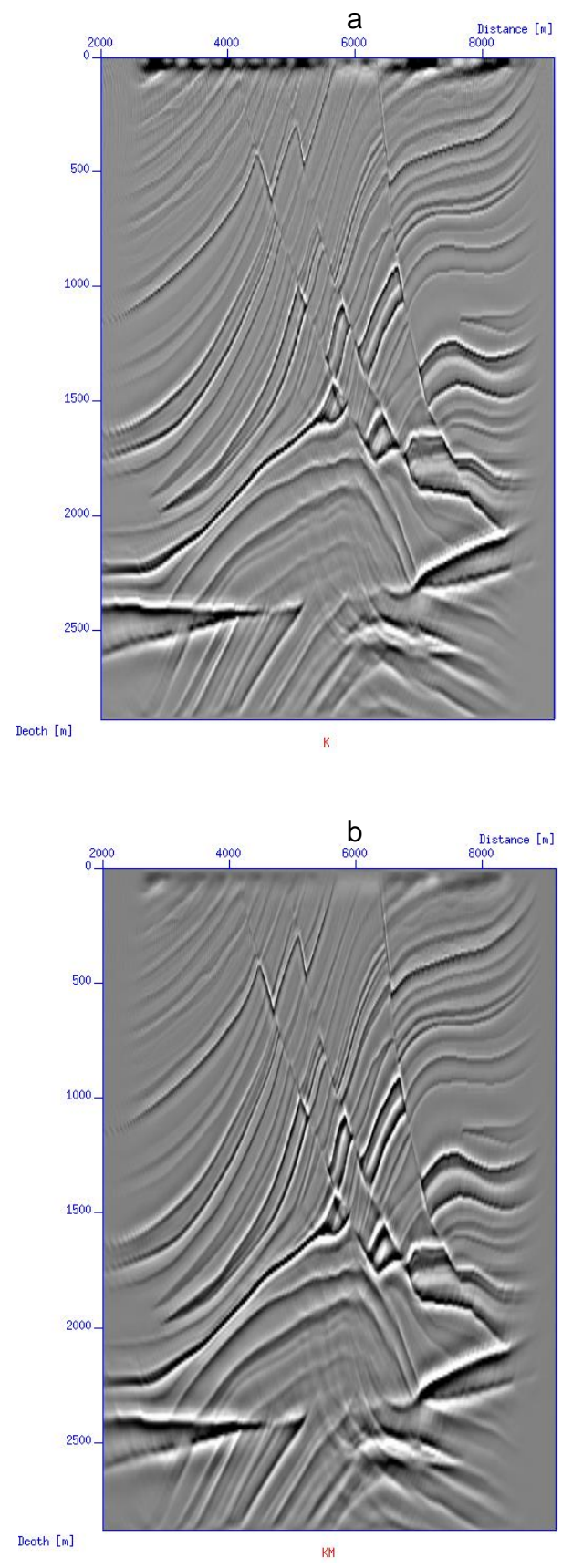

Figure 2 - Depth migrated sections resulting from the stacking of the migrated sections. Top: (a) K-PSDM. Bottom: (b) KM-PSDM. 

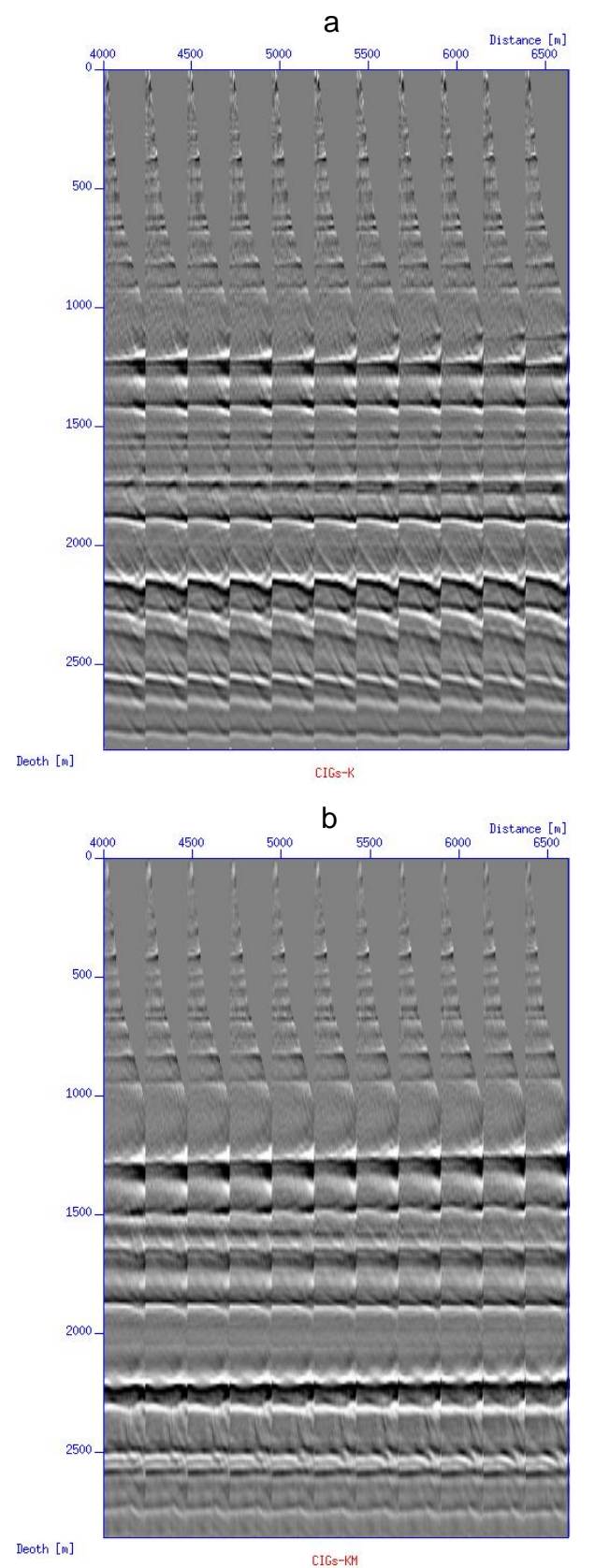

Figure 3 - Common image gathers (CIGs) resulting from the Marmousi synthetic data prestack depth migration. Top: (a) K-PSDM. Bottom: (b) KM-PSDM.

\section{Conclusions}

In this work we have introduced a 2D migration procedure known as modified true-amplitude 2D Kirchhoff migration (KM-PSDM).The present procedure is an integral operator that works in the kernel of the conventional Kirchhoff migration operator, over the seismic data, superposing paraxial reflections present in the vicinity of the reference trace and restricted to the projected Fresnel zone of seismic experiment. The KM-PSDM migration was tested in the synthetic common-offset Marmousi seismic data. The results show a noticeable enhancement in the resolution of the final migrated images when compared to images derived using the conventional Kirchhoff migration algorithm. Therefore, based on the present results shown in this paper, the KGB algorithm is an additional and attractive alternative tool for seismic imaging and interpretation, as well as another tool for the estimation of seismic amplitudes as hydrocarbon indicators.

\section{Acknowledgments}

Laboratory of Seismic Waves of the Institute of Geosciences.

\section{References}

Bleistein, N., 1987. On the imaging of reflectors in the Earth. Geophysics, 52, 931-942.

COSTA, M. J. S. Migração pré-empilhamento Kirchhoff feixes gaussianos $2.5 \mathrm{D}$ nos domínios afastamento comum e ângulo-comum., 2012. 114f. Tese (doutorado)Universidade Federal do Pará, Programa de PósGraduação em Geofísica, Belém, Pará, Brasil.

Ferreira, C.A.S., Cruz, J.C., 2005. Modified Kirchhoff prestack depth migration using the Gaussian beam operator as green function. 9th International Congress of the Brazilian Geophysical Society, Expanded Abstracts.

Ferreira, C. A. S. e Cruz, J. C. R., 2009. Gaussian beam modified true-amplitude diffraction stack migration: Application to Marmousi dataset. Expanded Abstracts of the 11th. SBGF international meeting,Salvador, Brasil.

Garabito, G., Stoffa, P. L., Ferreira, C. A. S., Cruz, J. C. R., 2012. Part II - CRS-beam PSDM: Kirchhoff-beam prestack depth migration using the 2D CRS stacking operator. Journal of Applied Gephysics, vol 85: 102-110.

Schleicher, J.; Tygel, M.; Hubral, P., 1993. 3D true amplitude finite - offset migration, Geophysics, vol 8:1112 $-1126$.

Schleicher, J.; Hubral, P.; Tygel, M.; Jaya, M. S., 1997. Minimun apertures and Fresnel zones in migration and demigration. Geophysics, 67, 183-194.

Schleicher, J.; Tygel, M.; Hubral, P., 2004. Trueamplitude seimic imaging: Monograph. Soc. Expl. Geophys. (Editor: B. Stolt).

Schneider, W. A., 1978. Integral formulation for migration in two and three dimensions. Geophysics, 43, 49-76. 
Sun, Y., Qin, F., Checkles, S., Leveille, J.P., 2000. 3-D prestack Kirchhoff beam migration for depth imaging. Geophysics 65, 1592-1603.

Tygel, M., Schleicher., J., Hubral, P., 1996. An unified approach to 3D seismic reflection imaging - Part II: Theory. Geophysics, vol 61:759-775. 